\title{
The origins of multifractality in financial time series and the effect of extreme events
}

\author{
Elena Green ${ }^{1, a}$, William Hanan ${ }^{1}$, and Daniel Heffernan ${ }^{1,2}$ \\ 1 Department of Mathematical Physics, National University of Ireland Maynooth, Maynooth, Co. Kildare, Ireland \\ 2 School of Theoretical Physics, Dublin Institute for Advanced Studies, 4 Dublin, Ireland
}

Received 27 January 2014 / Received in final form 31 March 2014

Published online 11 June 2014 - c EDP Sciences, Società Italiana di Fisica, Springer-Verlag 2014

\begin{abstract}
This paper presents the results of multifractal testing of two sets of financial data: daily data of the Dow Jones Industrial Average (DJIA) index and minutely data of the Euro Stoxx 50 index. Where multifractal scaling is found, the spectrum of scaling exponents is calculated via Multifractal Detrended Fluctuation Analysis. In both cases, further investigations reveal that the temporal correlations in the data are a more significant source of the multifractal scaling than are the distributions of the returns. It is also shown that the extreme events which make up the heavy tails of the distribution of the Euro Stoxx 50 $\log$ returns distort the scaling in the data set. The most extreme events are inimical to the scaling regime. This result is in contrast to previous findings that extreme events contribute to multifractality.
\end{abstract}

\section{Introduction}

Multifractal analysis has proved to be a valuable method of capturing the underlying scaling structure present in many types of systems via generalised dimensions [1] and $f(\alpha)$ spectra [2]. These systems include diffusion limited aggregation [3-5], fluid flow through random porous media [6], atomic spectra of rare-earth elements [7], clustercluster aggregation [8] and turbulent flow [9]. In physiology, multifractal structures have been found in heart rate variability [10] and brain dynamics [11], and multifractal analysis has been helpful in distinguishing between healthy and pathological patients [12]. Multifractal measures have also been found in man-made phenomena such as the Internet [13], art [14] and the stock market [15-17].

The concept of multifractality was first introduced in the context of turbulence. It was soon applied to finance because of its heavy tails and long-term dependence. These two features are also argued to be present in financial data $[18,19]$.

Performing multifractal analysis helps to increase our knowledge about the financial system and further characterise it. Many studies have found multifractal scaling in financial data [20-23]. An understanding of this multifractal structure can enable deeper understanding of the dynamics of financial markets. If it is found to be a universal feature of financial data, it provides an additional benchmark by which to measure the fitness of financial models. This in turn can help in the design of well performing port folios and in risk management [17].

\footnotetext{
a e-mail: elena.s.green@nuim.ie
}

The Multifractal Model of Asset Returns (MMAR) was introduced by Mandelbrot et al. [24] as an explanation of the volatility clusters in financial data and to include "outliers", large deviations which make up the fat tails of the return distribution. The MMAR was presented as an alternative to Autoregressive Conditional Heteroscedasticity (ARCH) models which were introduced by Engle [25] to account for volatility clustering. The MMAR incorporates fat tails, fractional Brownian motion $\mathrm{B}_{\mathrm{H}}{ }^{1}$ and the concept of "trading time" being distinct from physical time [24].

The main assumption of the MMAR is that the distinct trading time warps the financial time series into a multifractal structure. It takes the multifractality of the financial time series as a given. It also rejects the concept of outliers, insisting that even the most extreme events should be accounted for by a decent model. The results presented in this paper add credence to the assumption of multifractality as a stylised facts of financial data. However they also cast doubt on the inclusion of the most extreme events which was advocated by Mandelbrot and others [19].

Two distinct empirical data sets are examined in this paper. They are distinct in location and time scale. One is an American index with prices recorded daily (Dow Jones Industrial Average (DJIA)) and the other is a European index with prices recorded each minute (Euro Stoxx 50). The test for multifractality is carried out on the log returns

\footnotetext{
1 Where Brownian motion has Hurst exponent $H=1 / 2, B_{H}$ has Hurst exponent $\mathrm{H}, 0<\mathrm{H}<1$. $\mathrm{H}<1 / 2$ for an antipersistent process, $H>1 / 2$ for a persistent process. Brownian motion with $\mathrm{H}=1 / 2$ has no memory.
} 
which were constructed from the price time series by:

$$
Z(t)=\log (S(t+\Delta t))-\log (S(t))
$$

where $\mathrm{S}(\mathrm{t})$ is the price at time $\mathrm{t} . \Delta \mathrm{t}$ is one day for DJIA and one minute for Euro Stoxx 50.

The method used to find the scaling in the data is Multifractal Detrended Fluctuation Analysis (MF-DFA) [26]. The data and method are further described below.

The rest of this paper is laid out in the following way: Section 2 outlines the method used to uncover the multifractal structure of the data. Section 3 describes the empirical data and how the parameters of MF-DFA were set. Section 4 presents the results of the analysis. In Section 5 some further analysis is conducted to find the source of the multifractal structures found in the data. Finally Section 6 contains a summary and conclusions.

\section{Multifractal detrended fluctuation analysis}

There are a number of numerical methods by which to find the multifractal spectrum of time series. Two of the most well-known are the Wavelet Transform Modulus Maxima (WTMM) method [27,28] and Multifractal Detrended Fluctuation Analysis (MF-DFA) [26]. It has been shown that for data where the true fractal structure is unknown, MF-DFA is the recommended method of these two, showing less bias and being less likely to give a false positive result [29-31]. This is the method used in this paper.

MF-DFA is well suited to time series analysis because it is designed for data of a finite length $\mathrm{N}$, without requiring an $\mathrm{N} \rightarrow \infty$ approximation for validity [26]. Also this method treats the data simply as a one-dimensional line and assigns new values to each portion of the time series. This deals with the data having direction-dependent scaling properties and the nonequivalence of the time and value axes [26]. The assigned values are then assessed for multifractality.

The method involves the following steps, beginning with a disaggregated time series $X$ such as a set of financial log returns.

1. Transform $X$ into its mean-reduced cumulative sums $Y, Y_{j}=j_{i=1}^{j} X_{i}-\bar{X}$. This new data set is aggregated, resembling a random walk rather than a noise series, and has mean 0 .

2. Starting from the beginning, divide $Y$ into nonoverlapping segments of length $s$. Since $s$ may not divide evenly into $\mathrm{N}$, make another set of segments starting at the end of the data and coming back so that no piece of the data is left out. This results in $2[\mathrm{~N} / \mathrm{s}]=2 \mathrm{~N}_{\mathrm{s}}$ boxes covering the entire data set. Find the least-squares polynomial fit $y_{v}$ of order $m$ to the data in each segment $\mathrm{v}=1, \ldots, 2 \mathrm{~N}_{\mathrm{s}}$.

3. Find the root-mean-square error or fluctuation between the fit and the data in each segment. This is the value $F^{2}(v, s)$ of segment $v$ of size $s$;

$$
\mathrm{F}^{2}(\mathrm{v}, \mathrm{s})=\frac{1}{\mathrm{~s}}_{\mathrm{i}=1}^{\mathrm{s}}\left(\mathrm{Y}[(\mathrm{v}-1) \mathrm{s}+\mathrm{i}]-\mathrm{y}_{\mathrm{v}}[\mathrm{i}]\right)^{2}
$$

for $\mathrm{v}=1, \ldots, \mathrm{N}_{\mathrm{s}}$ and

$$
\begin{aligned}
& \mathrm{F}^{2}(\mathrm{v}, \mathrm{s})=\frac{1}{\mathrm{~s}}_{\mathrm{i}=1}^{\mathrm{s}}\left(\mathrm{Y}\left[\mathrm{N}-\left(\mathrm{v}-\mathrm{N}_{\mathrm{s}}\right) \mathrm{s}+\mathrm{i}\right] \ldots\right. \\
& \left.\ldots-y_{v}[i]\right)^{2}
\end{aligned}
$$

for $\mathrm{v}=\mathrm{N}_{\mathrm{s}}+1, \ldots, 2 \mathrm{~N}_{\mathrm{s}}$.

4. Introduce a parameter $\mathrm{q}$. Find the $\mathrm{qth}$ order variance $F_{q}$ for a range of both positive and negative $q$ for each segment size $\mathbf{S}$.

$$
F_{q}(s)={\frac{1}{2 N_{s}}}_{v=1}^{2 N_{s}} F^{2}(v, s)^{q / 2}
$$

For $\mathrm{q}=0$, use the quenched average $\mathrm{F}_{0}(\mathrm{~s})=$ $\exp \left[\frac{1}{4 N_{s}} \quad \begin{array}{ll}2 N_{s} \\ v=1\end{array} \ln \left(F^{2}(v, s)\right)\right]$.

5. Repeat steps 2, 3 and 4 for different segment lengths $\mathrm{s}$, finding a new set of values $F_{q}(s)$ in each case.

6. For each value of $q$, plot $F_{q}(s)$ versus $s$ on a doubly logarithmic scaled graph and find the least-squares linear fit to each curve. If an appropriate linear region (more than one order of magnitude of $s$ ) is found for all values of $q$, it can be concluded that there is scaling in the data and the slopes $h(q)$ can be calculated. If $h(q)$ varies with $q$, one can conclude that the scaling is multifractal.

7. Find the multifractal exponent $\mathrm{T}(\mathrm{q})$,

$$
\mathrm{T}(\mathrm{q})=\mathrm{qh}(\mathrm{q})-1-\mathrm{qH}
$$

where $\mathrm{H}=\mathrm{h}(1)-1$ is called the nonconservation parameter ${ }^{2}$ and proceed to the $f(\alpha)$ spectrum via the Legendre transforms:

$$
\begin{aligned}
\alpha(q) & =\frac{d \tau(q)}{d q} \\
f(\alpha(q)) & =\alpha(q) q-T(q) .
\end{aligned}
$$

A plot of $f(\alpha)$ versus $\alpha$ is the multifractal spectrum for the time series data $X$.

Multifractality has been reported in cases where there is only the spurious scaling which can arise in non- or monofractal time series [31,34-36], and so caution is required. It is critically important to check the linearity of the logarithmic plots as described in Step 6. Plotting the slope of the line over a moving window should reveal roughly constant slope over the length of the line before linearity is accepted. Oscillation about a straight line is

${ }^{2}$ This is an adjustment to the original definition of $\mathrm{T}$ given by Kantelhardt et al. [26], $\mathrm{T}(\mathrm{q})=\mathrm{qh}(\mathrm{q})-1$. It accounts for the fact that $F^{2}(v, s)$ is not strictly speaking a measure on the time series $Y$. For further details, see references $[32,33]$. 
Table 1. Summary statistics for the log return data examined in this paper for multifractal properties. $N$ is the sample size of the data, $\mu$ is the sample mean and $\sigma$ the sample standard deviation. $H$ is the estimated Hurst exponent of the sample.

\begin{tabular}{cccccccccc}
\hline Data & $\Delta \mathrm{t}$ & $\mathrm{N}$ & Min & Max & 川 & $\sigma$ & Skewness & Kurtosis & $\mathrm{H}$ \\
\hline DJIA & 1 day & 20922 & -0.2563 & 0.1427 & $1.89 \times 10^{-4}$ & 0.0117 & -0.5931 & 27.2784 & 0.5146 \\
Euro Stoxx 50 & 1 min & 109545 & -0.0935 & 0.0610 & $-4.5257 \times 10^{-6}$ & 0.0011 & -2.1397 & $1.0335 \times 10^{3}$ & 0.448 \\
\hline
\end{tabular}
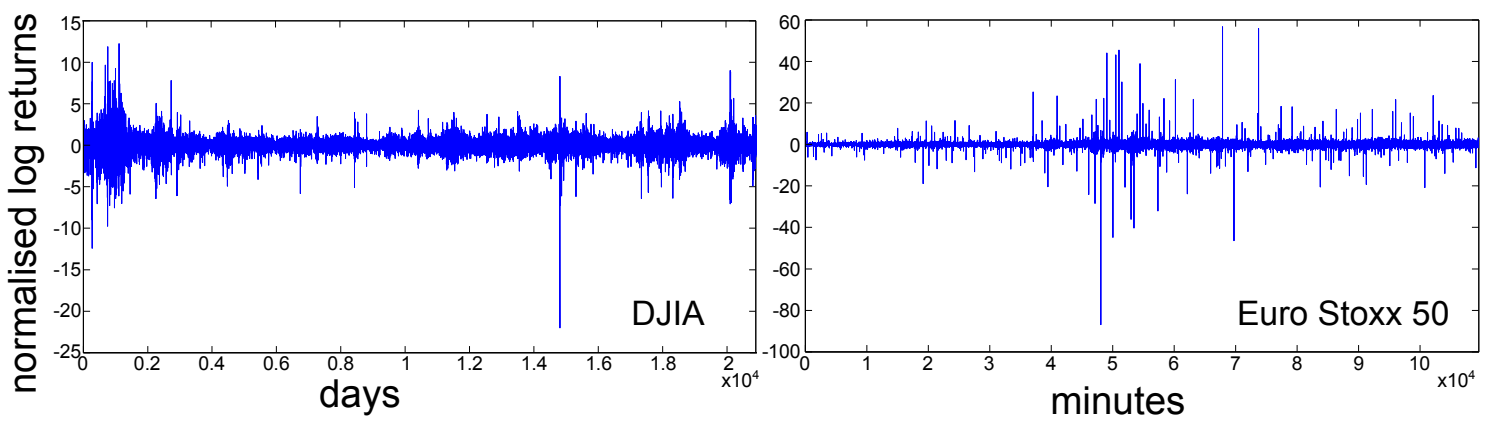

Fig. 1. Graph of the daily log return data of the DJIA and of the minutely log return data of Euro Stoxx 50 whose multifractal properties are examined in this paper. The log returns are given in units of standard deviation for ease of comparison in this figure.

to be expected as these are statistical fractals. However, if there is no significant linear region revealed by the local slopes, we cannot conclude that there is multifractal scaling in the data.

Finite-size effects are also an important consideration [35]. Short monofractal time series can appear multifractal due to linear correlations. Since the log return data considered here have negligible linear correlations (Hurst exponent $H \approx 1 / 2$, see Tab. 1), this is not a concern for our analysis.

Multiscaling Multifractal Analysis [37], an extension to the MF-DFA method, has recently been recommended to pick up information from any cross-overs that might be in the data. A crossover is a point where the slopes change on the graph of $\log \left(F_{q}\right)$ vs. $\log (s)$. Since we see no such crossover points in our data, there is no need for this extra analysis.

\section{Data and implementation}

The first data examined is the daily log returns of the DJIA from 1928 to 2012 which contains 20922 points. This is a weighted average of the prices of 30 companies based in the United States. Its normalised form is shown in Figure 1. The dramatic downturn of late 2007 and 2008 is included in this data set and the major "Black Monday" crash of October 19th 1987 is obvious at approximately $1.5 \times 10^{4}$ days.

The Dow Jones Euro Stoxx 50 was also examined and the normalised log returns for the time period of interest are also shown in Figure 1. This is an index of 50 Blue-chip sector leaders from 12 Eurozone countries which was launched in 1998. The data is minutely and runs for a year, from the start of May 2008 until the end of April 2009. There are 109545 points in this time series. The high volatility that can be seen in the middle of the time series corresponds to the time around the Lehman Brother's collapse in September 2008.

The MF-DFA method was applied to both log return time series. Summary statistics for the log return data of DJIA and Euro Stoxx 50 are presented in Table 1. The exclusion of overnight returns in the minutely time series made no difference to the results of the analysis and so they have been retained. All time outside of trading hours has been omitted.

For the implementation of MF-DFA, certain parameters have to be chosen. Both data sets were detrended by order $\mathrm{m}=1$ polynomials as this led to the best scaling results. The length scale $s$ takes small steps from a minimum of 10 to a maximum of $\mathrm{N} / 4=\mathrm{N}_{4}$, where $\mathrm{N}$ is the length of the time series. This means that at the largest scale there are 8 boxes since there are $2 N_{s}$ boxes for each $\mathrm{S}$. This range of scales is proposed by Kantelhardt et al. [26].

A wide range of 1001 equally spaced values of the parameter $q$ was chosen, with $q \in[-50,50]$. This is a very wide range in comparison with other studies [12,20,26,38] where it is standard to use $q \in[-5,5]$. However, for smaller ranges of $q$, less of the multifractal spectrum is revealed. It is found that $f(\alpha) \approx 0$ for the examined data as $q \rightarrow \pm 50$, and this captures the full spectrum.

\section{Results}

The plots of $F_{q}(s)$ versus $s$ on a doubly logarithmic scale for the DJIA data for selected values of $q$ are shown in Figure 2a. Although 1001 values of q were used in the analysis, it is not practical to show all of them on the graph. The segment size $s$ takes 59 values from 10 to 5230 . By checking the local slopes of these lines (Fig. 2b) it is possible to identify a scaling region over more than two orders of magnitude from $s=10$ to $s=2000$. This region of scaling was then used to construct the multifractal spectrum which is displayed in Figure 3. 


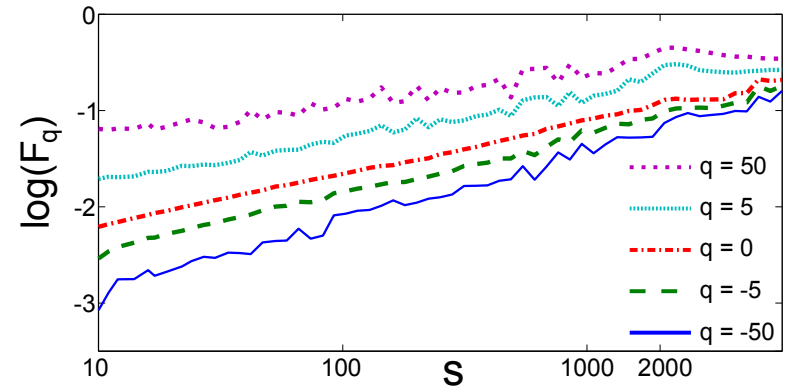

(a)

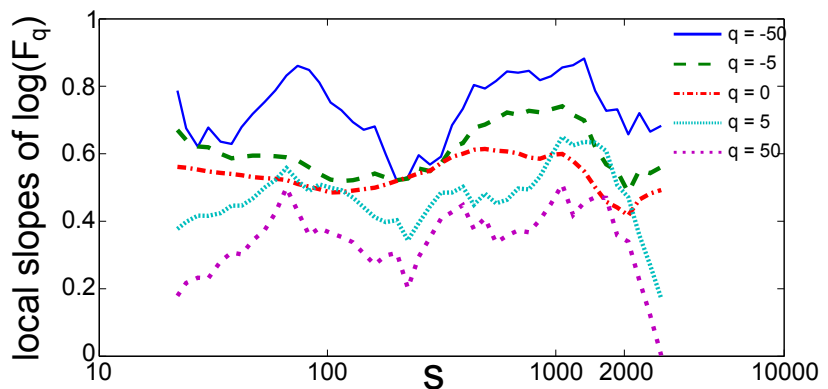

(b)

Fig. 2. DJIA: (a) Graph of $\log \left(F_{q}\right)$ versus $\log (\mathbf{s})$ for selected values of $q$ as shown on the graph. (b) Graph of the local slopes of the lines in (a) calculated over 15 points for the same values of $q$. The slopes remain reasonably constant for $s \in[10,2000]$.

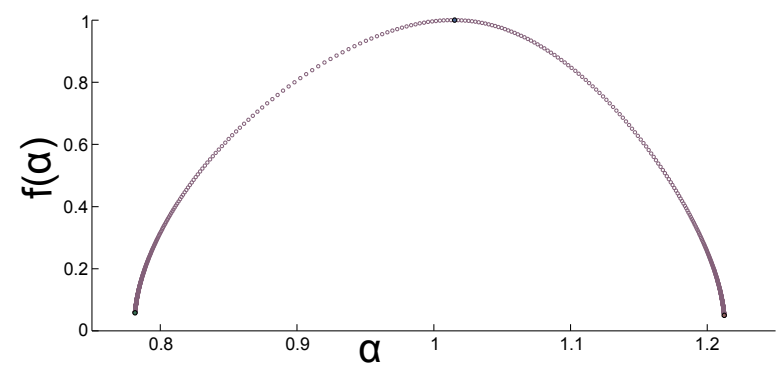

Fig. 3. Graph of the multifractal spectrum, $f(\alpha)$ versus $\alpha$, for DJIA calculated for the length scales $s \in[10,2000]$ and with $q \in[-50,50]$

The results of the initial check for scaling for the Euro Stoxx 50 data are shown in Figure 4. It is not obvious whether or not there is scaling in this data. The slopes are not of the quality of those for DJIA observed in Figure $2 b$. The multifractality is less certain in this case. It could be argued that the local slopes in Figure 4a are not constant over a sufficient range of $s$ and so indicate a lack of scaling in the Euro Stoxx 50 data. In this case, this data could be presented as a counterexample to the stylised fact of the presence of multifractality in financial return data [39].

It could also be argued that scaling is present over more than two orders of magnitude; for $65 \quad s \leq 10000$. It breaks down for small segment sizes (s 65) when $q$ is negative. The abrupt change in $F_{q}(s)$ can be explained by the presence of a section of consecutive zeroes in the log returns. Since $F$ is a measure of the distance of the data

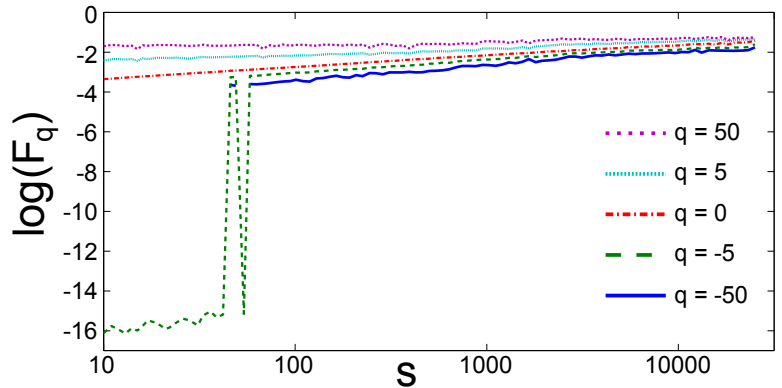

(a)

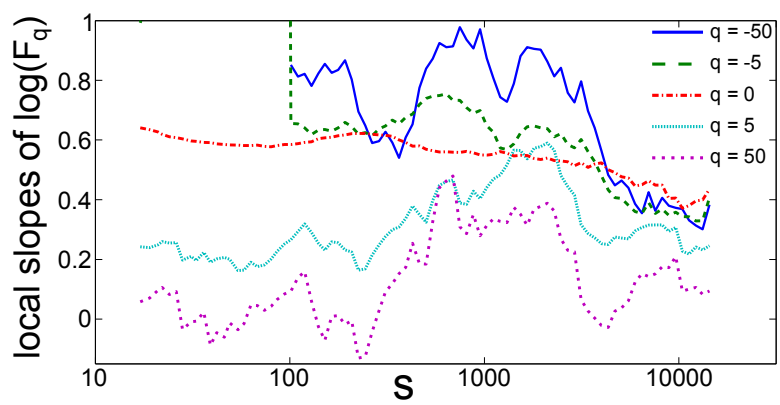

(b)

Fig. 4. Euro Stoxx 50: (a) Graph of $\log \left(F_{q}\right)$ versus $\log (s)$ for selected values of $q$ as shown on the graph. (b) Graph of the local slopes of the lines in (a) calculated over 15 points for the same values of $q$.

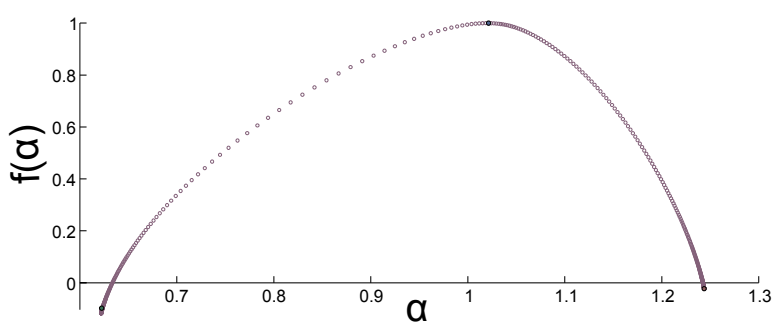

Fig. 5. Graph of the multifractal spectrum, $f(\alpha)$ versus $\alpha$, for Euro Stoxx 50 calculated for the length scales $s \in[65,10000]$ and with $q \in[-50,50]$.

in any segment from a linear fit, when a segment $v$ lies within this interval of zeroes, $F(v)$ is close to zero. The smallest $F$ dominates in $F_{q}$ when $q<0$ which explains the drop in $\log \left(F_{q}\right)$ as $S$ decreases for $q<0$.

The multifractal spectrum for the range $65 \mathrm{~s} \leq$ 10000 is shown in Figure 5. The left side of the spectrum is stretched out and $f(\alpha)<0$ for $\alpha \quad 0.63$. The left side represents the areas of high $F_{q}$ and so this is evidence of poor scaling, and possibly even a breakdown in scaling, of the most volatile segments. As we shall show in Section 5.2, it is the extreme return events which are responsible for these phenomena.

The fact that Figure 4 seems to indicate a lack of scaling and yet the spectrum in Figure 5 can still be produced shows that real caution is required when conducting multifractal analysis. A wide smooth spectrum does not imply that the data actually has multifractal scaling. 
Table 2. Summary of the main results of MF-DFA on the daily DJIA and minutely Euro Stoxx 50 data for a range of values of $q \in[-50,50]$. The truncated data has extreme events replaced with smaller ones. It is discussed in Section 5.2. Here $\Delta \alpha=\alpha_{\max }-\alpha_{\min }$.

\begin{tabular}{cccccccc}
\hline Data & $\mathrm{f}(-50)$ & $\mathrm{\alpha}(-50)$ & $\mathrm{f}(0)$ & $\alpha(0)$ & $\mathrm{f}(50)$ & $\alpha(50)$ & $\Delta \alpha$ \\
\hline DJIA & 0.049882 & 1.2124 & 1 & 1.0126 & 0.058373 & 0.78155 & 0.43087 \\
Euro Stoxx 50 & -0.023382 & 1.2437 & 1 & 1.0184 & -0.0981 & 0.6226 & 0.62162 \\
Truncated Euro Stoxx 50, c = 15 & -0.021838 & 1.265 & 1 & 1.0169 & 0.017184 & 0.78068 & 0.48431 \\
\hline
\end{tabular}

The spectra in this paper seem shifted to the right in comparison to those in the literature [20,23,38]. This can be accounted for by the updated definition of $\mathrm{T}(\mathrm{q})$ in Step 7 in Section 2. A summary of the results of MF-DFA for both data sets is contained in Table 2 .

\section{The origins of multifractality}

It is generally accepted that there are two possible sources of multifractal scaling in time series data [26]. It could be predominantly due to (1) the long-term correlations of small and large fluctuations or (2) the data being drawn from a heavy-tailed probability distribution. Both of these influences can individually be removed from the data to reveal what impact they have on the multifractality of the time series.

Other recent work has shown that multifractality can be viewed as the result of the Tweedie Convergence Theorem, similarly to how Gaussian noise can be seen as the result of the Central Limit Theorem [40,41]. However, since financial time series are not sequences of independent identically distributed random variables, the convergence theorem does not apply. Here we will examine the traditional sources: correlations and the shape of the distribution.

\subsection{Source of scaling - correlations}

A simple way to check if correlations in the data produce any scaling is to shuffle the data as suggested by Kantelhardt et al. [26]. Shuffl ing removes time correlations and any scaling that remains must be due to the probability distribution from which the data is drawn. The distribution of the values is not affected by reordering the series.

Any individual shuffle may still contain some correlations, so to be sure to completely rid the data of all correlations, both the DJIA and the Euro Stoxx 50 data were shuffled 100 times, each random permutation beginning with a new random number generator seed in MatLab. The function $F_{q}$ was found for each of the shuffled data sets. These were then averaged to find $\overline{F_{q}(s)}=$ $\frac{1}{100} \quad{ }_{i=1}^{100} F_{q, i}(s)$, where the index $i$ identifies the shuffled data sequence. The doubly logarithmic plots of $\overline{F_{q}(s)}$ versus $S$ for different $q$ were then checked for linearity. The results are shown in Figures 6 and 7. The same analysis was conducted with the quenched average, $\overline{\log \left(\mathrm{F}_{\mathrm{q}}(\mathrm{s})\right)}$, with very similar results.

For both time series, there is no significant linear region in the plots of $\log \left(\overline{F_{q}(s)}\right)$ versus $\log (\mathrm{s})$. Thus we do

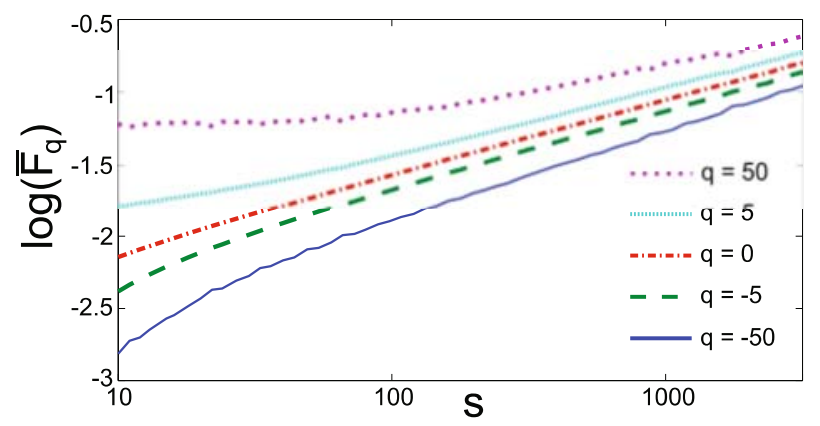

(a)

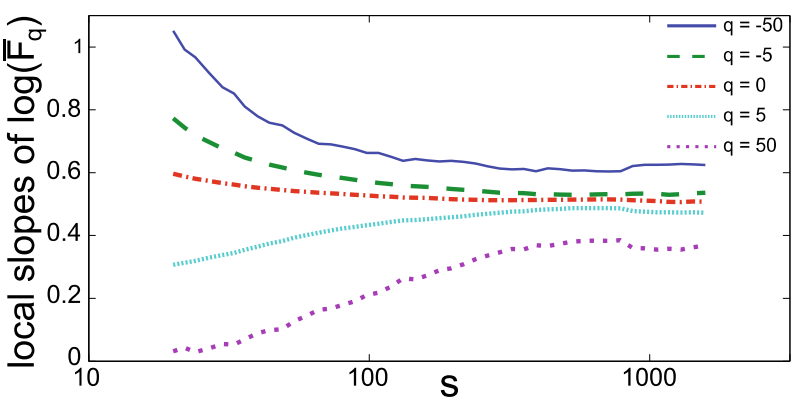

(b)

Fig. 6. Shuffled DJIA data: (a) Graph of the log of the averaged scaling function, $\log \left(\overline{F_{q}}\right)$, versus the $\log$ of the scale, $\log (\mathrm{s})$, for selected values of $q$ as shown on the graph. (b) Graph of the local slopes of the lines in (a) calculated over 15 points for the same values of $q$.

not have the rationale to proceed to calculate $h(q)$ and must instead conclude that multifractal scaling is absent in these shuffled data sets.

Other studies $[16,20,22,42,43]$ have found multifractal scaling in shuffl ed financial data. However, as no explicit investigation of the logarithmic plots and their local slopes was conducted, the conclusion that multifractal scaling is present is not justified.

Different degrees of shuffling were also employed so that correlations of different length scales can be removed [31]. Rather than reordering every point in the data, the data was divided into intervals of length I. Then each set of I adjoining points were kept together while the order of the intervals was shuffled. This helps to reveal how robust the scaling is to the presence of temporal correlations.

The result of this analysis for the DJIA data is shown in Figure 8. Intervals of lengths $\mathrm{I}=10,50$, $100,500,1000$, and 5000 were kept intact and only the 


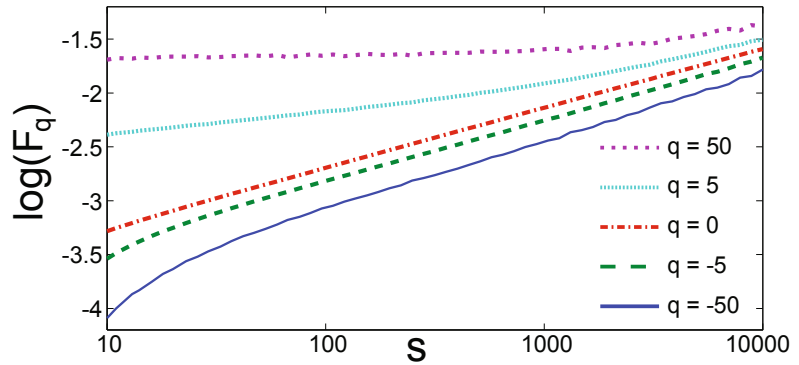

(a)

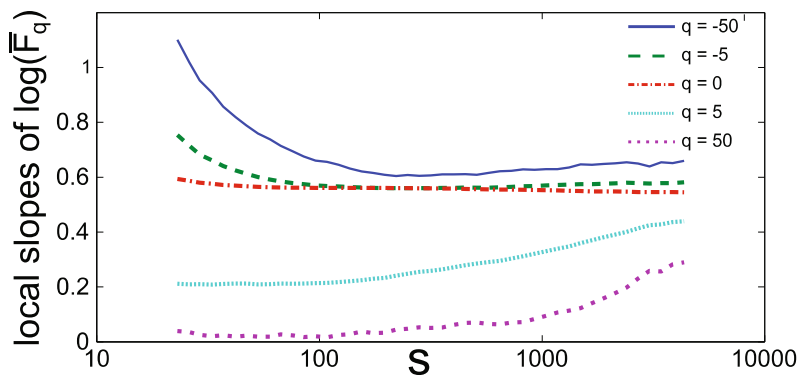

(b)

Fig. 7. Shuffled Euro Stoxx 50 data: (a) Graph of the log of the average scaling function, $\log \left(\overline{F_{q}}\right)$, versus the $\log$ of the scale, $\log (\mathrm{s})$, for selected values of $q$ as shown on the graph (b) Graph of the local slopes of the lines in (a) calculated over 15 points for the same values of $q$.

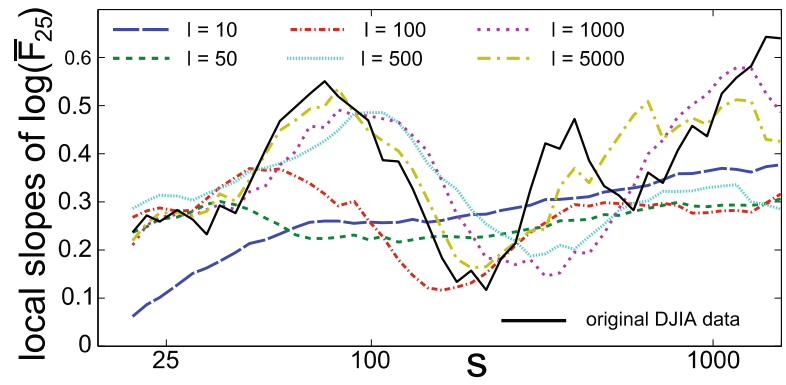

Fig. 8. DJIA: Graph of the local slopes of $\log \left(\overline{F_{25}}\right)$ versus $\log (\mathrm{s})$ for shuffles with various interval lengths I as indicated on the graph. The slope for the original data is also shown, corresponding to the lines in Figure $2 b$.

order of the intervals was rearranged randomly 100 times as was done for the original shuffling. Then the plots of $\log \left(\overline{F_{q}}\right)$ versus $\log (\mathrm{s})$ were found. Figure 8 shows the slopes of these plots for $q=25$.

The scaling is worst for the shortest interval length, $\mathrm{I}=10$ for which any temporal correlations longer than 10 days have been destroyed. The local slopes for the scaling function is strongly increasing with the box size rather than oscillating about a constant value. For longer intervals, more memory is preserved and correspondingly the scaling improves.

When $I=500$, the scaling is preserved. At this length and for longer intervals, the slopes are oscillating about a constant and do not show sustained curvature in a single direction. This value, $I=500$, gives an indication of the

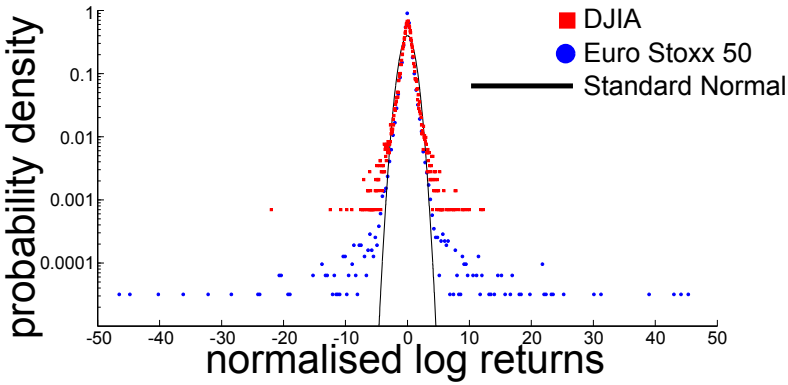

Fig. 9. Graph of the normalised empirically found distribution of the DJIA (red squares) and Euro Stoxx 50 (blue circles) data along with the Standard Normal curve for comparison. It is shown on a semi-logarithmic scale to make the fat tails clear.

length of temporal correlations that are significant to the multifractal scaling in the data. It is possible to infer that the data has memory to the order of 100's of days. The scaling does not survive a more substantial change in the ordering of the data.

Linear correlations in the log-returns only live for a time scale of a few minutes, but the correlation in the absolute values or the squared returns last much longer [18]. The results presented in this section provide evidence that long-term correlations, which are removed by the shuff ing procedure, are a major source of the multifractality in both the DJIA daily data and the Euro Stoxx 50 minutely data.

\subsection{Source of scaling - distribution}

The empirical distribution of financial log returns is generally found to be leptokurtic [18]. Figure 9 shows the distribution for both the daily DJIA data and the minutely Euro Stoxx 50 data. They have been normalised and are shown along with the Standard Normal curve for comparison on a semi-logarithmic scale. The Euro Stoxx 50 data is not shown completely; a negative log return of $-86 \sigma$ and some positive returns of around $60 \sigma$ are cut off to make the graph clearer. The most extreme event in the DJIA data is Black Monday, 19th October 1987. It constituted a drop of over $22 \sigma$ for this index.

The effect of the distribution on the scaling in the time series can be revealed in a number of ways [20]. One method involves truncating the tails of the distribution. If large positive and negative log returns are replaced by less extreme ones, the data will retain its temporal correlations while removing the fat tails of the distribution. This truncated data can then be tested for multifractality to see what influence the tails of the distribution have on the $f(\alpha)$ spectrum.

In order to carry out this analysis, any log returns $z$ in the data which satisfy $|z|>c \sigma$ were replaced by $\operatorname{sgn}(z) c \sigma$ where $\sigma$ is the standard deviation of the raw data and $c$ is the truncation point. $c$ varies from 1 to 10 for DJIA and 1 to 15 for Euro Stoxx 50. The usual analysis was then conducted on this new data set to find the scaling properties. The number of points which were truncated 
Table 3. The cumulative frequency table showing the number of log returns whose absolute value is larger than the given truncation point $\mathrm{c}$ for both the DJIA and Euro Stoxx 50 time series.

\begin{tabular}{cccccccccccccccc}
\hline C: & 1 & 2 & 3 & 4 & 5 & 6 & 7 & 8 & 9 & 10 & 11 & 12 & 13 & 14 & 15 \\
\hline DJIA & 3875 & 957 & 362 & 162 & 75 & 45 & 27 & 13 & 10 & 5 & 4 & 3 & 1 & 1 & 1 \\
Euro Stoxx 50 & 14166 & 2516 & 671 & 299 & 206 & 161 & 128 & 109 & 90 & 79 & 68 & 57 & 53 & 45 & 42 \\
\hline
\end{tabular}

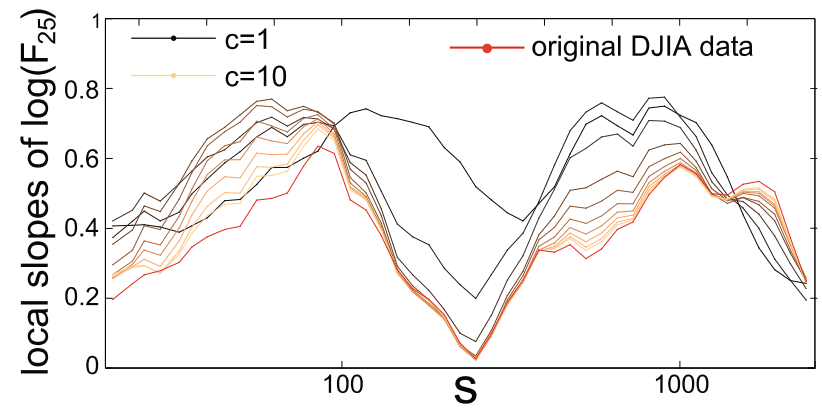

(a)

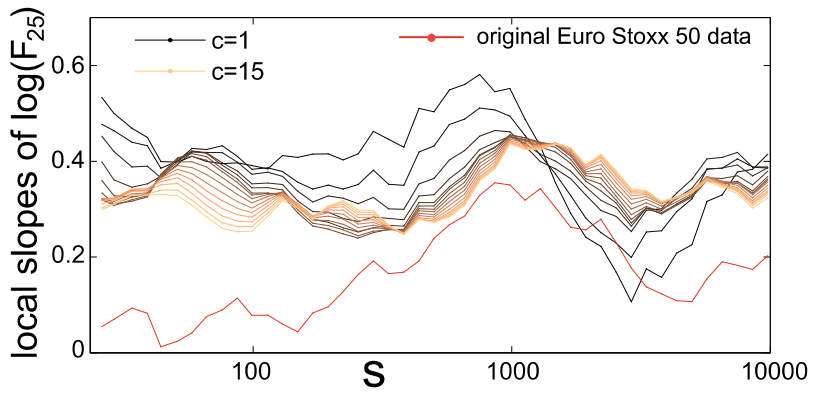

(b)

Fig. 10. (a) Graph of the local slopes calculated over 15 points of $\log \left(F_{25}\right)$ versus $\log (\mathrm{s})$ for DJIA $\log$ returns for a range of truncation points $c \sigma$ for the values of $c$ indicated on the graph. (b) Graph of the local slopes calculated over 15 points of $\log \left(\mathrm{F}_{25}\right)$ versus $\log (\mathrm{s})$ for the Euro Stoxx $50 \log$ returns for a range of truncation points $c \sigma$ for the values of $c$ indicated on the graph. The slope for the original data in both cases is the thick red line.

for each level $\mathrm{C}$ are shown in Table 3. The local slopes of the plots of $\log \left(F_{q}\right)$ versus $\log (s)$ for $q=25$ for both the DJIA and Euro Stoxx 50 are shown in Figure 10.

For the DJIA data shown in Figure 10a, the oscillations of the slopes become more severe for more severe truncation. However the scaling is preserved. Extreme events are evidently not imperative to the scaling in this time series.

For the Euro Stoxx 50 data in Figure 10b, the scaling is actually improved after modest truncation. Apart from the most severe cases of $c=1$ and $c=2$, the slopes are reasonably constant. The severe leptokurtosis of the Euro Stoxx 50 log returns is actually a hindrance to the scaling. This was initially indicated by the stretched left-hand side of its $f(\alpha)$ plot shown in Figure 5. This can be contrasted with the much more symmetric $f(\alpha)$ plot for the Euro Stoxx 50 data truncated at $c=15$ in Figure 11 .

This spectrum is narrower than that of the original Euro Stoxx 50 data $(\Delta \alpha=0.48$ here compared to 0.62 for the original, see Tab. 2). This result is in agreement with

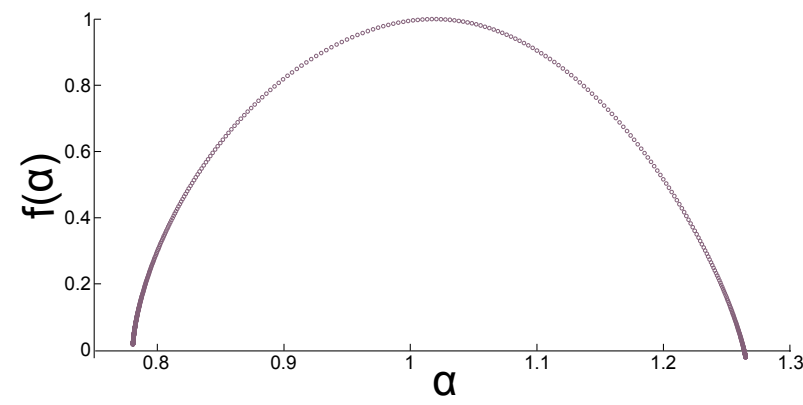

Fig. 11. Graph of the multifractal spectrum, $f(\alpha)$ versus $\alpha$, for the Euro Stoxx 50 data after it has been truncated so that any $\log$ returns $|z|>15 \sigma$ have been replaced by $z=\operatorname{sgn}(z) 15 \sigma$. It has been constructed for $s \in[65,10000]$.

others which have found that the multifractal spectrum narrows when extreme events are truncated [20,44]. This is to be expected as the narrower range of $\alpha$ reflects the reduced heterogeneity in the data. However, where others $[20,35,43-46]$ have used the spectrum width $\Delta \alpha$ as a metric for the level of multifractality, we have conducted a more detailed analysis. We inspect the plots of $\log \left(F_{\mathrm{q}}\right)$ and the produced $f(\alpha)$ spectrum, giving more insight into the effect of the extreme events. The extreme events cause the spectrum to become asymmetric and negative at one end. The left side of the spectrum in Figure 5 is stretched due to poor statistics in those extreme areas of the time series. Therefore the spectrum width $\Delta \alpha$ is unreliable in this case to base conclusions on about the strength of multifractal scaling.

Some studies have found that extreme events cannot simply be thought of as scaled-up versions of smaller events [47-49]. Extreme events appear to be drawn from a different distribution and do not scale well with more modest returns. The results of the analysis of Euro Stoxx 50 lend some support to this idea. While the scaling in the complete data set is uncertain, the scaling improves when large positive and negative returns are removed. This indicates that they may belong to a separate scaling regime or they may not scale at all. However the number of extreme events is too small to test them separately for scaling.

In general there is no consensus in the published literature as to whether it is the fat tails of the distribution or the temporal correlations which contribute most to the multifractal scaling in financial data. It has been found that distribution contributes more to the multifractal scaling than do the temporal correlations in some daily data [20,22]. Others have shown evidence of the opposite [50] or that both sources are significantly present [43]. Work on higher-frequency data $[23,45]$ has found that the correlations are the most likely cause of 
multifractality. Mixed results have been found for foreign exchange rates $[46,51,52]$. These varied results imply that the main source of multifractality is dependent on the particulars of each specific data set and that there is no universal law.

The resolution of the time series has an impact on the results of MF-DFA. At small resolutions (e.g. minute) where returns are more highly leptokurtic, the extreme events can distort the scaling, while such distortion is absent at larger resolutions (such as days). The 42 most extreme points removed from the Euro Stoxx 50 time series by the truncation method have a major impact on the scaling results. The data appears to made up of a multifractal subset and these outliers.

\section{Conclusions}

A systematic study has been carried out on the multifractal properties of two financial time series: daily DJIA $\log$ returns from 1928 to 2012 and minutely Euro Stoxx $50 \mathrm{log}$ returns from 2008 to 2009. Multifractal scaling has been found in the DJIA data. Careful attention was paid to finding an appropriate linear region in the logarithmic plots of the scaling function $F_{q}$ versus the segment size $s$ before concluding that multifractal scaling is present and proceeding to the plot of $f(\alpha)$ versus $\alpha$. This examination makes this study more comprehensive than many others which have investigated financial data for multifractality.

The scaling is open to interpretation for the Euro Stoxx 50 time series. The uncertainty illustrates the need for caution and for further analysis techniques to be developed in this area. A set tolerance of linearity of the $\log \left(F_{q}\right)$ versus $\log (\mathrm{s})$ plots is required within which multifractality can be accepted.

In the case of the DJIA daily data, the multifractal spectrum is nearly exactly symmetric. The one for the minutely Euro Stoxx 50 data, on the other hand, is stretched on the left and $f(\alpha)<0$ for $\alpha$ 0.63. The shape of its asymmetric $f(\alpha)$ plot reveals that the extremely volatile areas of the data scale differently from the rest of the data or do not scale at all.

The temporal correlations in both data sets have been shown to be a significant source of the multifractal scaling. The scaling does not survive in either time series when the data is reordered, thereby removing correlations.

Adjustments were also made to the distribution of the returns to reveal its effect on the scaling. The results indicate that the extreme events do not conform to the scaling law which is followed by the smaller returns. In the case of Euro Stoxx 50, the scaling is improved when the most extreme events are removed. This is consistent with the asymmetrical shape of its multifractal spectrum.

Our results are more comprehensive than others as we are not content with the single metric $\Delta \alpha$ to measure the level of multifractal scaling present in the data. We conduct a more comprehensive examination, including the $\log \left(F_{q}\right)$ plots, their local slopes, and the $f(\alpha)$ spectrum. This more detailed analysis leads us to different conclusions than those presented in other studies. We conclude that these extreme events are actually inimical to the multifractal scaling in the Euro Stoxx $50 \log$ returns.

We would like to thank our referees for bringing some relevant papers to our attention. We also thank Sepanda Pouryahya for helpful conversations. Elena Green and Daniel Heffernan acknowledge the support of Science Foundation Ireland under Grant number 08/ SRC/ FM1389.

\section{References}

1. P. Grassberger, Phys. Lett. A 97, 227 (1983)

2. T.C. Halsey, M.H. Jensen, L.P. Kadanoff, I. Procaccia, B.I. Shraiman, Phys. Rev. A 33, 1141 (1986)

3. T.C. Halsey, P. Meakin, I. Procaccia, Phys. Rev. Lett. 56, 854 (1986)

4. W.G. Hanan, D.M. Heffernan, Phys. Rev. E 85, 021407 (2012)

5. W.G. Hanan, D.M. Heffernan, Phys. Rev. E 77, 011405 (2008)

6. H.E. Stanley, P. Meakin, Nature 335, 405 (1988)

7. A. Cummings, G. O’Sullivan, W.G. Hanan, D.M. Heffernan, J. Phys. B 34, 2547 (2001)

8. W.G. Hanan, D.M. Heffernan, J.C. Earnshaw, Chaos Solit ons Fractals 9, 875 (1998)

9. B.B. Mandelbrot, J. Fluid Mech. 62, 331 (1974)

10. P. Ivanov, L. Amaral, A. Goldberger, S. Havlin, M. Rosenblum, Z. Struzik, H. Stanley, Nature 399, 461 (1999)

11. Y. Zheng, J. Gao, J.C. Sanchez, J.C. Principe, M.S. Okun, Phys. Lett. A 344, 253 (2005)

12. E.A. Ihlen, Front. Physio. 3, 141 (2012)

13. A. Feldmann, A.C. Gilbert, W. Willinger, SIGCOMM Comput. Commun. Rev. 28, 42 (1998)

14. N. Sala, in Thinking in Patterns: Fractals and Related Phenomena in Nature, edited by M.M. Novak (World Scientific, Singapore, 2004)

15. B.B. Mandelbrot, R. Hudson, On the (mis) Behaviour of Markets, a Fractal View of Risk, Ruin and Reward (Profile Books, London, 2005)

16. L. Czarnecki, D. Grech, Acta Physica Polonica A 117, 623 (2010)

17. A. Turiel, C.J. Pérez-Vicente, Physica A 322, 629 (2003)

18. R. Cont, Quant. Finance 1, 223 (2001)

19. B.B. Mandelbrot, in Thinking in Patterns: Fractals and Related Phenomena in Nature, edited by M.M. Novak (World Scientific, Singapore, 2004)

20. W.X. Zhou, Europhys. Lett. 88, 28004 (2009)

21. V. Romanov, V. Slepov, M. Badrina, A. Federyakov, in Computational Finance and Its Applications III, edited by M. Constantino, M. Larran, C.A. Brebbia (WIT Press, 2008), pp. 13-22

22. K. Matia, Y. Ashkenazy, H.E. Stanley, Europhys. Lett. 61 , $422(2003)$

23. P. Suárez-García, D. Gómez-Ullate, Physica A 394, 226 (2014)

24. B.B. Mandelbrot, A.J. Fisher, L.E. Calvet, A Multifractal Model of Asset Returns (Cowles Foundation, 1997)

25. R.F. Engle, Econometrica 50, 987 (1982)

26. J.W. Kantelhardt, S.A. Zschiegner, E. Koscielny-Bunde, S. Havlin, A. Bunde, H.E. Stanley, Physica A 316, 87 (2002)

27. J.-F. Muzy, E. Bacry, A. Arneodo, Int. J. Bifurc. Chaos 4, $245(1994)$ 
28. J.-F. Muzy, E. Bacry, A. Arneodo, Phys. Rev. Lett. 67, 3515 (1991)

29. P. Oświęcimka, J. Kwapień, S. Drożdż, Phys. Rev. E 74, 016103 (2006)

30. P. Oświẹcimka, J. Kwapień, S. Drożdż, R. Rak, Acta Physica Polonica B 36, 2447 (2005)

31. A.Y. Schumann, J.W. Kantelhardt, Physica A 390, 2637 (2011)

32. Z. Yu, L. Yee, Y. Zu-Guo, Chin. Phys. B 20, 090507 (2011)

33. P. Jizba, J. Korbel, Methods and techniques for multifractal spectrum estimation in financial time series, in Proceedings ASMDA, 2013

34. T. Lux, Int. J. Mod. Phys. C 15, 481 (2004)

35. W.X. Zhou, Chaos Solitons Fractals 45, 147 (2012)

36. J.-P. Bouchaud, M. Potters, M. Meyer, Eur. Phys. J. B 13, 595 (2000)

37. J. Gieralt owski, J.J. Żebrowski, R. Baranowski, Phys. Rev. E 85, 021915 (2012)

38. J.R. Thompson, Analysis of Market Returns Using Multifractal Time Series and Agent-Based Simulation, Ph.D. thesis, Raleigh, 2013
39. M. Segnon, T. Lux, Multifractal models in finance: Their origin, properties, and applications, Technical report, Kiel Working Paper, 2013

40. W.S. Kendal, Physica A 401, 22 (2014)

41. W.S. Kendal, B. Jørgensen, Phys. Rev. E 84, 066120 (2011)

42. J. Barunik, T. Aste, T. Di Matteo, R. Liu, Physica A 391, 4234 (2012)

43. S. Kumar, N. Deo, Physica A 388, 1593 (2009)

44. G. Oh, C. Eom, S. Havlin, W.S. Jung, F. Wang, H.E. Stanley, S. Kim, Eur. Phys. J. B 85, 214 (2012)

45. J. Kwapień et al., Physica A 350, 466 (2005)

46. Y. Wang, C. Wu, Z. Pan, Physica A 390, 3512 (2011)

47. D. Sornette, Phys. Rep. 378, 1 (2003)

48. V.S. L'vov, A. Pomyalov, I. Procaccia, Phys. Rev. E 63, 056118 (2001)

49. D. Sornette, Int. J. Terraspace Sci. Eng. 2, 1 (2009)

50. S. Benbachir, M. El Alaoui, Int. Res. J. Finance Econ. 78, 6 (2011)

51. P. Norouzzadeh, B. Rahmani, Physica A 367, 328 (2006)

52. H. Chen, C. Wu, Physica A 390, 2926 (2011) 
